\title{
Habitat structure and fish: assessing the role of habitat complexity for fish using a small, semiportable, 3-D underwater observatory
}

\author{
Philipp Fischer ${ }^{1}$, Arnd Weber, Georg Heine, and Harald Weber \\ University Konstanz, Limnological Institute, 78457 Konstanz, Germany \\ ${ }^{1}$ Present address: Alfred-Wegener-Institute/Biologische Anstalt Helgoland, Kurpromenade 201, 27498 Helgoland, Germany
}

\begin{abstract}
In contrast to terrestrial ecology, three-dimensional (3-D) imaging technology is not well established as a method for studying species-habitat interactions in aquatic ecology. In this study, we used a semi-portable, digital, 3-D underwater observatory designed for long-term exposure in shallow water habitats to assess fish-habitat interactions to artificial structures of different complexity. The observatory was mounted on a cable-car system and was moved along a $50 \mathrm{~m}$ transect parallel to five artificial structures of different complexity and one control site. The optical unit took high-resolution digital images, in pairs, from each structure and the control site at a frequency of $30 \mathrm{~min}$ over $24 \mathrm{~h}$ and was then moved remotely to the next structure. The system was operated for 2 mo without being recovered and took a total of 2160 stereoscopic image pairs. The system proved highly valuable in assessing small-scale temporal patterns in fish-habitat interactions, as well as changes in habitat preferences of fish over the diel cycle. Such small-scale temporal and spatial patterns are important for a detailed understanding of specieshabitat interactions, not only in fish but also in most aquatic macro-organisms. Nevertheless, such patterns can hardly be assessed using classical sampling devices due to labor and animal protection restrictions. However, the possibility of a significant increase in quantity of sampling through the use of remote imaging technology is accompanied by a significant increase in labor costs for image analysis. Therefore, further progress in semi- and fully automated systems for image analysis is needed, including 3-D imaging tools, for ecological studies.
\end{abstract}

The degree of structural complexity of an aquatic habitat is an important proximate factor for its attractiveness to fish. Structural complexity of habitats has been shown to significantly affect the temporal-spatial distribution of fish (Bohnsack and Sutherland 1985; Crowder and Cooper 1982; Fischer 2000, 2004), their somatic growth and survival (Johnson et al. 1988; Persson and Eklöv 1995), as well as the individual foraging efficiency and capture rate both of prey and predator fish (Manatunge et al. 2000; Priyadarshana et al. 2001; Weaver et al. 1997).

Besides these direct effects on individual organisms, habitat structure is also known to increase spawning success and food availability (Kuznetsov 1970), improve egg survival (Welcomme and Cowx 1998), and increase the density of lower trophic level organisms, thus improving food availability and composition (Crowder and Cooper 1982; Diehl and Kornijow 1998).

\footnotetext{
Acknowledgments

We thank the technical engineering department of University Konstanz for assembling the prototype of the 3-D observatory, several student helpers for the image analysis, and two unknown reviewers for helpful and welcomed comments on the manuscript. This work was supported by the German Research Foundation within the Special Collaborative Project SFB 454 'Littoral of Lake Constance.'
}

Although the importance of habitat complexity for aquatic communities is unquestionable, quantitative assessments of the attractiveness of different levels of habitat complexity are difficult to conduct. This is mainly because sampling complex structures is challenging. When using classical net-based sampling gears, such as gill-nets or fyke-nets, in structurally complex habitats, the risk of gear damage, or even gear loss, is high. In contrast, when adequate sampling techniques are available, such as enclosure nets or pop-up nets (Larson and Johnson 1986), sampling structurally complex habitats is often timeconsuming and leads to a low number of samples per time unit, and therefore, lower significance. Furthermore, most enclosure or pop-up net devices rely on the removal of the entire structure to sample its associated fish community and are thus highly destructive.

Other, nondestructive assessment methods such as SCUBA counting (Basset 1994; Johnson and Lynch 1992; Rogers and Bergersen 1999) fish telemetry, even though less invasive, are relatively time-consuming and provide only temporally or numerically restricted data. Due to these limitations, these methods often provide inconsistent results due to individual gear-specific peculiarities and restrictions, especially with regard to the temporal use of a certain habitat structure (Larson and Johnson 1986). 
In a recent study on the problems of sampling complex habitats, Pratt and colleagues (2005) discussed the use of advanced, in situ imaging techniques, and corresponding image sampling protocols, for the assessment of habitat-fish relationships in structurally complex habitats. In the critical discussion of this study, the authors addressed the fact that it is often not possible to resolve species-habitat relationships at a sufficiently fine scale to adequately address the temporalspatial factors in fish-habitat relationships, even when using state-of-the-art underwater video technology. Pratt and colleagues (2005) blamed this on the comparatively restricted field of view of most video camera systems, too short filming durations, or the low number of freeze-frame sub-samples taken in most studies. These limiting factors, especially of diver-operated in situ imaging technologies, often lead to extremely high variability in organism counts per frame with too many zero counts, especially when the target organisms are mobile. This leads to a dramatic loss of statistical power in the subsequent data analysis.

Due to the above limitations of sampling frequency within a certain time period on the one side and a sufficiently long survey duration on the other side, we developed a low-cost, portable three-dimensional (3-D) underwater observatory (subsequently called "RemOS-1 = Remote Optical System 1") to overcome these restrictions. Our main objectives for this system were for it (i) to be comparatively lightweight and easy to expose, especially in shallow water habitats, (ii) to be robust enough to withstand "normal" wind- or ship-induced hydrodynamic action in shallow water areas so it could be exposed for a (theoretically) unrestricted period, and (iii) to be fully controlled by a landbased control site via underwater cable and W-LAN technology to assess and process the taken 3-D images online without recovering the system from the water. Because sampling frequency (stereoscopic image pairs) would be significantly increased by the use of this system, we developed a simple and easy-to-use image analysis tool for analyzing the underwater 3-D image pairs produced by this system.

The system is able to take high resolution stereo digital photographs (up to 7 Million pixels) at a frequency of up to one stereo-pair per two minutes over $24 \mathrm{~h}$ at a maximum distance of about $1000 \mathrm{~m}$ from a land-based control unit. This image frequency and shoreline distance was determined to be sufficient for our application and easily realized within the options of standard cable data-transmission technology (RS424). Higher image frequencies as well as longer transmission distances to the shoreline would have been possible by using a fiber-optic cable connection providing greater bandwidth. However, because one goal of the project was to develop a straightforward, easy-to-use, and affordable system, and because we did not see a basic necessity for a higher bandwidth or image frequency for our scientific question, we renounced the implementation of fiber-optic transmission technology at this state of the project.
To assess the applicability of this observatory system for the analysis of fish-habitat relationships in shallow water habitats, we set up five artificial structures of different complexity in the littoral zone of Lake Constance, Germany at about $100 \mathrm{~m}$ from land in $2.5 \mathrm{~m}$ water depth. We then continuously assessed the associated fish community throughout $24 \mathrm{~h}$ for $1.5 \mathrm{mo}$ by stereo photography. To achieve this, the observatory was exposed for $24 \mathrm{~h}$ in front of one structure and took stereoscopic image pairs every $30 \mathrm{~min}$. The observatory was then automatically moved to the next structure by an underwater cable-car system, and again it took stereoscopic image pairs every $30 \mathrm{~min}$ for $24 \mathrm{~h}$, and so on. Based on these 48 stereo image pairs per $24 \mathrm{~h}$, the fish community associated with the different structures was analyzed with respect to fish species composition, fish density per $\mathrm{m}^{3}$, length-frequency distribution, and the main swimming direction of the fish. For the latter analysis, we used four categories: "swimming toward the pelagic zone," "swimming toward the littoral zone," "swimming parallel to the shoreline, lefthand (looking toward the shoreline)," "swimming parallel to the shoreline, righthand (looking toward the shoreline)."

Based on this data, we discuss the usability, advantages, and possible further progress in the use of this low-cost, in situ remote optic observatory technology to assess habitat-community relationships in complex shallow water habitats.

\section{Materials and procedures}

Artificial structures-All artificial structures used in the study had an identical base structure (Fig. 1a): a central vertical $1 \mathrm{~m}$ long polyethylene (PE) pillar $(\varnothing 60 \mathrm{~mm})$, which was fixed by a $0.8 \mathrm{~m}$ tent-peg-like steel rod in the sediment and held vertical by a small overhead floating device of about $0.2 \mathrm{~kg}$ buoyancy. Circles of 12, 8, and 4 identical PE rods (each $40 \mathrm{~cm}$ long, $\varnothing 4 \mathrm{~mm}$ ) subsequently called $1 / 1,1 / 2$, and $1 / 3$ density, were mounted on the central PE tube (Fig. 1b) at a longitudinal distance of $100 \mathrm{~mm}$ (Fig. 1a). This allowed for defined increasing complexity of the structures while keeping the base design of each observatory identical. Additionally, one "overhead shading" structure was provided by a single PE plate (80 $\times 80 \mathrm{~cm}$ ), floating $100 \mathrm{~cm}$ from the bottom, to analyze the effects of "only overhead cover," and finally, a $50 \times 50 \mathrm{~cm}$ base dimension patch of submerged aquatic vegetation (Potamogeton perfoliatus), collected from a nearby naturally growing patch, was used as natural structure. As a control site, an empty area without any structures was marked with a tent peg. Therefore, five different structures and one control site were used in the experiment.

All structures were installed 3 weeks prior to the start of the data acquisition proper parallel to the shoreline at about $100 \mathrm{~m}$ offshore, in $2.5 \mathrm{~m}$ water depth, close to the transition zone between the littoral and the adjacent slope area. The distance between the individual structures was $5 \mathrm{~m}$, and the location of each along the transect was chosen randomly using a six-sided dice. The sediment in this area was homogenous and com- 


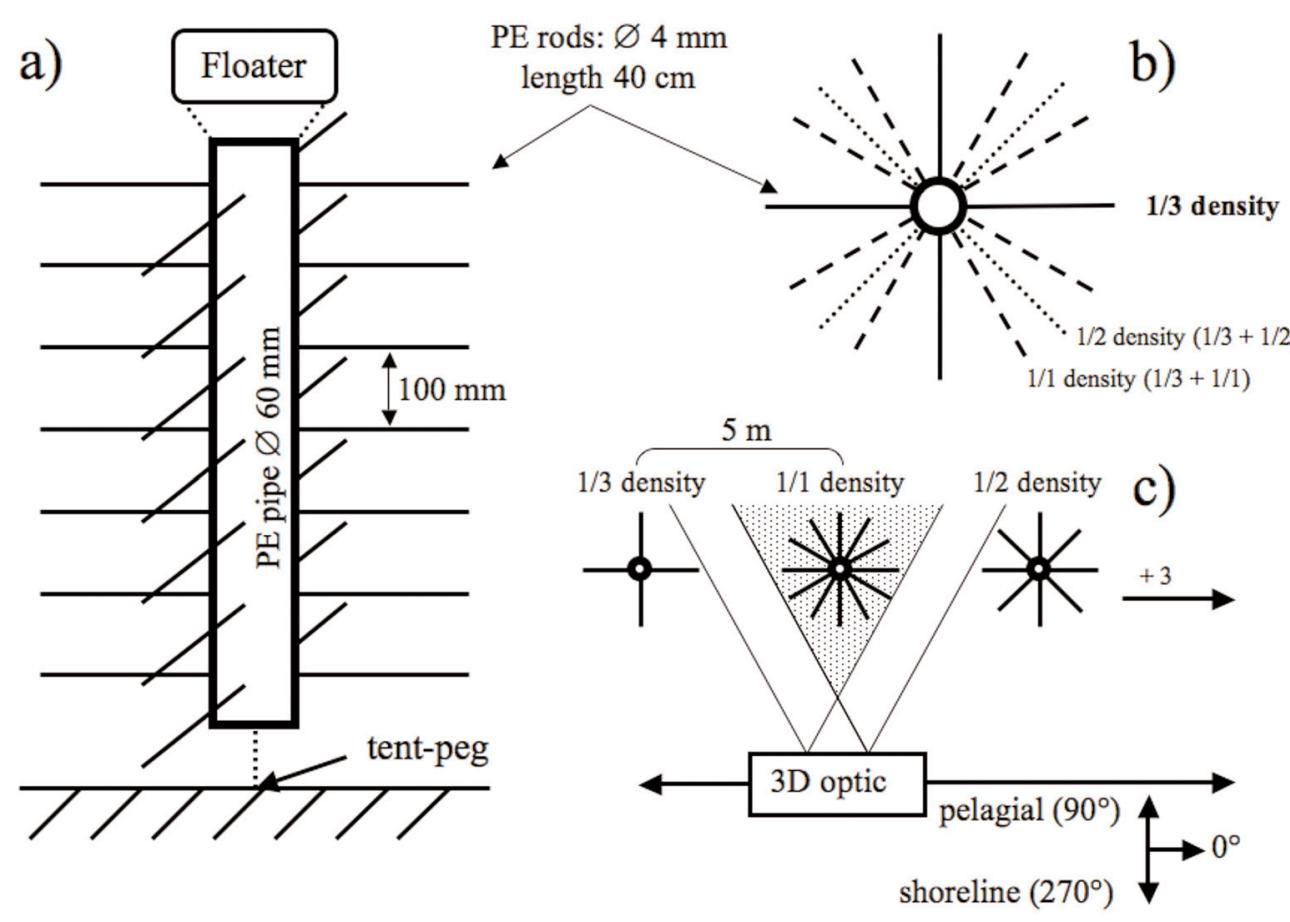

Fig. 1. The experimental set-up. (a) Artificial structure $(1 / 3$ density) with four $P E$ rods $(40 \mathrm{~cm}$ long) arranged circular to the central rod at longitudinal distances of $10 \mathrm{~cm}$, (b) circular arrangement of the PE rods. Density $1 / 1=$ solid and dashed rods, density $1 / 2=$ solid and dotted rods, density $1 / 3=$ solid rods only, (c) in situ arrangement of the experimental set-up. The three structures at 1/3,1/1, and $1 / 2$ density are arranged from left to right followed by $P$. perfoliatus (not shown), shading and control structures. (indicated by an arrow to the right and the +3 (structure) sign (for a detailed description of the five and control structures, see text). The field of optical overlap of the 2-digital photo cameras is indicated as the lightly shaded area. The orientation of the optical perspective of the system is shown in degrees with $0^{\circ}$ looking parallel to the shoreline to the right.

posed of sand and clay with low sediment structure at all sites.

The underwater observatory-The stereo-optical unit consists of 2-digital consumer cameras (CANON Power-Shot G6, image sensor type 1/1.8-inch CCD, effective pixel 7.1 Mio.) with additional wide converter (CANON WC-DC58N conversion factor 0.7). The actual focal length of the camera system is $5.0 \mathrm{~mm}$. Both cameras were mounted in a watertight housing of stainless steel (Fig. 2), which was connected to a land-based control site via a $200 \mathrm{~m}$ long (max. $1000 \mathrm{~m}$ ) underwater cable. Within the housing, the cameras are placed side by side on a rack at a horizontal distance of $120 \mathrm{~mm}$. A specifically designed microprocessor board in the housing allows simultaneous triggering of both cameras as well as downloading of both pictures sequentially to an external storage medium at the land-based control unit.

Camera settings-The focus mode of each camera is set to "manual" to avoid problems with auto-focus adjustments especially at night. In this study, the system was focused on a target distance of $1.5 \mathrm{~m}$ where a picture overlap of $83 \%$ was reached.

The aperture and exposure time of both cameras is set to "automatic mode." Because pictures are taken continuously over 24 h, a TTL flash (CANON Speed light 550X, "TTL operation mode") was connected to the right camera. This flash was set to forced mode to ensure identical illumination con- ditions for all images over the diel cycle. This set-up significantly facilitates the subsequent analysis of the stereo image pairs due to a constant image exposure.

To allow exact synchronization of the two cameras (an

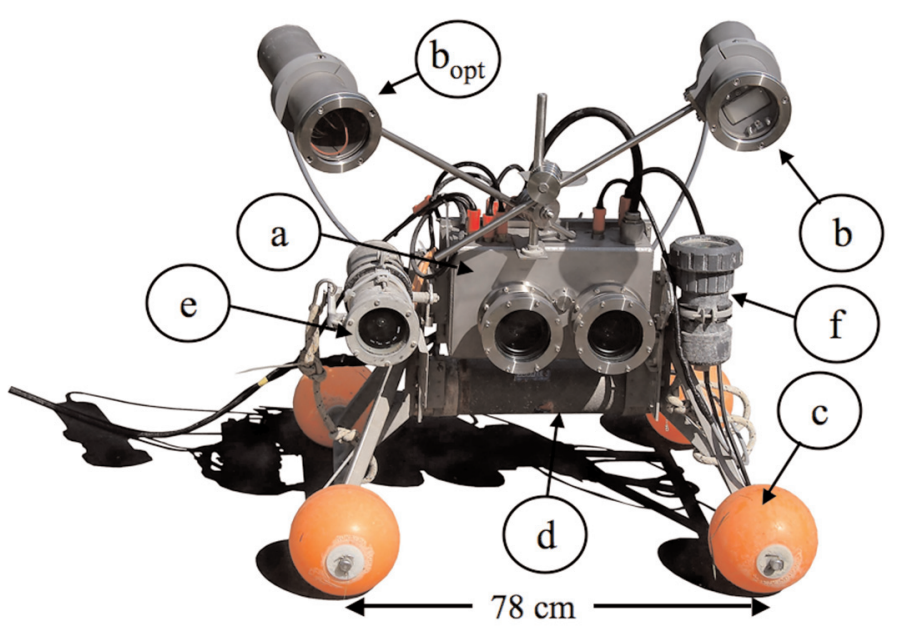

Fig. 2. An annotated photograph showing the major components of the RemOs1 underwater observatory: (a) watertight housing, (b) flash housing (the second flash housing shown on the left $\left[b_{\text {opt }}\right]$ is for an optional second flash light), (c) wheel-balls (deep-sea buoyancy balls), (d) batterypack, (e) Web-cam housing, (f) temperature and light sensor housing. 
indispensable prerequisite for an exact stereoscopic image analysis of moving objects), a software time-shift option is included in the microprocessor board, allowing the shift of trigger signal for the left camera in 1 ms steps. To exactly synchronize both cameras, the trigger signal for the left camera is temporally shifted relative to the right camera until both images are correctly exposed. Because in TTL operation mode, image exposure is fully controlled by the intensity and duration of (in our system) a single flash illumination phase and not by the duration of the opening phase of the aperture of the camera, a full synchronization of the left (nonflashing) camera and the right (flashing) camera is ensured when both images are correctly exposed. Only then is it certain that the single and comparatively short flash illumination phase $(<1 \mathrm{~ms})$ triggered by the right camera is placed temporally correctly in the approximately $10 \mathrm{~ms}$ lasting shutter opening phases of both cameras. With the above-described camera and flash light settings, less than $2 \%$ of the stereo image pairs taken over the entire experimental period were incorrectly exposed and rejected from further analysis. This happened mainly due to yet unknown system failures when, e.g., one camera could not be activated at all or got hung up during the camera acceleration process prior to image acquisition. Because both cameras are completely shut down and powered off after each image acquisition, these exceptional system failures are overcome at the next activation phase.

The underwater unit of the observatory is connected by a standard polyvinyl chloride [PVC] cable $(16 \times 1.5 \mathrm{~mm}$ PVC) to a land-based, autonomous, control computer (consumer personal computer with Microsoft Windows 2000 operating system installed in a watertight $40 \times 60 \times 30$ aluminum box on the shoreline). The cable connection is used for (i) power supply to the underwater unit ( $24 \mathrm{~V}$ direct current) and (ii) for data transfer between the underwater unit and the land-based control unit via an RS424 protocol. Additionally, the shorelinebased control unit is connected via standard WLAN technology to the Limnological Institute of the University of Konstanz (distance about $2 \mathrm{~km}$ ) and can be remotely accessed by the consumer remote-control software, Carbon Copy Vers. 5.60 (Altris 2001). This configuration allows for continuous operation and remote maintenance of both the land-based control unit and the underwater unit without further field handling. Furthermore, it allows an online download, image control, and quality adjustment of the pictures, as well as for a regular backup of the images to avoid data loss in case of a system breakdown. The latter happened twice during the study period, once because a hacker broke into the system and caused severe software damage, and a second time because of a breakdown in the underwater energy supply, which is designed to operate the system for about $6 \mathrm{~h}$ without any external power supply.

The routine power consumption of the entire underwater unit is about $600 \mathrm{~mA}$. During image acquisition and data transfer, the power consumption is increased to about $900 \mathrm{~mA}$.
A pick-load of up to $1.4 \mathrm{~A}$ is reached for about $15 \mathrm{~s}$ after flash illumination, when the condenser of the flash has to be recharged.

Data acquisition-To sequentially assess the fish assemblages associated with the different structures, the system was moved every $24 \mathrm{~h}$ to another (randomly selected) structure (Fig. 1c). This was done by a remotely controlled underwater cable-car transportation system by which the underwater unit could be freely moved along a $50 \mathrm{~m}$ transect parallel to the structures. The exact positioning of the stereo-unit in front of a certain structure was done manually, using an integrated video system (Fig. 2e), that allows an online view of the optical perspective of the stereo-photo cameras from a central control unit at the Limnological Institute by remote access. The entire system (the stereo-optical unit and the cable car transportation system) was installed 10 days before the assessment itself started.

This sampling design allowed us to assess each structure and the control site every seventh day. The assessment itself was run for a total of 2 mo from 10 August 2004 to 6 October 2004, yielding a total of eight 24-h sampling days per structure, with $6 \mathrm{~d}$ intervals between two consecutive 24 -h observations.

In addition to the stereo-optical unit, the underwater unit was equipped with sensors for temperature and light (temp.: \pm $0.1^{\circ} \mathrm{C}$; light: $\pm 0.01 \log \operatorname{lum} \mathrm{x} \mathrm{m}^{2}$ ) with a sampling frequency of once per minute over $24 \mathrm{~h}$ (Fig. 2f).

Calibration of the 3-D optic-To assess the accuracy and precision (Kellner et al. 2004) of the optical system in terms of size measurements, calibration experiments were carried out according to the established procedures of analog stereo photography (Cook 1986; Klimley 1980; Rebikoff 1984).

To assess measurement accuracy (deviation between the measured size of an object and its real size), objects of known length were exposed in the water, in front of the stereo camera system at distances of 50,100,150, 200, and $250 \mathrm{~cm}$ in four orientations: parallel to the optical chip plane $\left(0^{\circ}\right)$, with inclination angles of $30^{\circ}$ and $60^{\circ}$ from the chip plane, and perpendicular to the chip plane (inclination angle $90^{\circ}$, parallel to the optical axis). Within each of these orientations, the object was adjusted to four different positions, $0^{\circ}$ (horizontal), $30^{\circ}$, $60^{\circ}$, and $90^{\circ}$ (vertical). By this method, 36 accuracy measurements was performed for one camera-object distance.

To determine the measurement precision (the system related measurement variation of the accuracy), each accuracy estimate was done ten times in sequence. The variation of these measurements were then calculated as coefficient of variation of the accuracy and reflect the precision of the system.

In contrast to analog stereoscopic photography, where the parallelism of the two optical axes of a pair of cameras has to be estimated by calibration pictures of an object at infinity (Cook 1986; Klimley 1980; Rebikoff 1984) and later corrected during image processing, in digital photography this can be done prior to the measurements by adjusting the two cameras on the mounting rack to an object at infinity. To do this in the system 


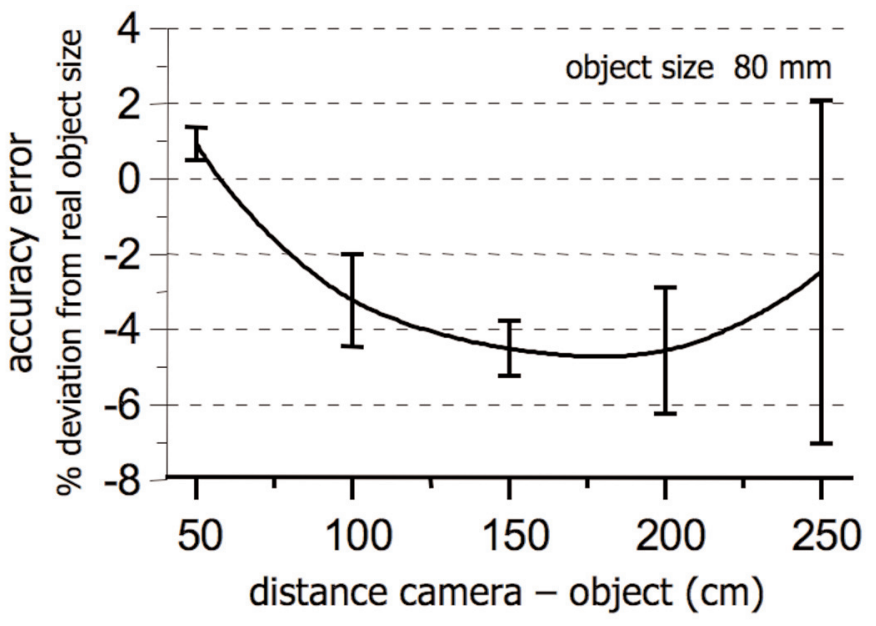

Fig. 3. Accuracy values (percent deviation between the measured size of an object and its actual size $[=100 \%]$ ) with increasing camera-object distance. Shown are mean values (connected by spline interpolation) with error bars based on 10 replicate measurements per distance. The error bars indicate the precision of the accuracy estimates and are calculated as coefficient of variation of the each accuracy value $(n=10)$.

used here, the two cameras were mounted in the housing on two separate platforms, that could be adjusted by micrometerscrews until each camera provided a picture in which an object at infinity is located at the identical position in terms of pixel distance from the upper and left edges of the image. Performing this camera adjustment before measurements meant that postmeasurement correction for non-parallelism of the two optical axes of the stereo cameras, a procedure that makes quantitative 3-D image analysis with analog systems more complicated (Boisclair 1991), becomes redundant.

Image analysis-To analyze the digital stereoscopic image pairs, the software package "RemSoft1" was developed in C++ at the University of Konstanz, Department of Technical Engineering. Using the stereo calculation algorithms of Klimley (1980), we adapted the algorithm for digital image analysis to give a pixel-based determination of the $x$ and $y$ coordinates of an identical object on both pictures of a digital stereoscopic pair of identical size. For object measurement, a pair of stereo images is loaded into the program in two separate frames, between which one can easily switch by pressing a previously assigned control key. An object (e.g., fish length) is then measured by first clicking with the mouse pointer on the tip of the fish's head and then at the desired measuring point for its length on the left image (e.g., fork length measuring point on the caudal fin), and then on the tip of the same fish's head and length measuring point on the right image. The software then automatically asks for the fish species measured, calculates the length and its orientation in the horizontal plane in $1^{\circ}$ steps counterclockwise from the optical axis, which is here assigned to $90^{\circ}$.

During this study, the software package did not enforce epipolar constraint technology for improved position sensing on the two images. However, this software enhancement is in progress for the next software release.
The software stores all the data, including the pixel-based $x$ and $y$ coordinates of the objects in the two pictures, in a tabdelimited ASCII worksheet, together with the filenames of the stereoscopic pair of pictures, the name of the measured object (e.g., fish species) and additional comments. This allows for further subsequent object analysis, e.g., the absolute distances of the fish to each other within a shoal and further distance related measurements. The software is designed for the operating system Microsoft Windows and is available upon request from the authors.

\section{Assessment}

System calibration-The calibration revealed an increased accuracy error (deviation between the measured and the real size of an object) with increased camera-object distance (Fig. 3). The system generally tends to underestimate the real length of an object with a maximal error of $-4.7 \%$ (the measured size is $4.7 \%$ smaller than the real size) at a camera-object distance of $170 \mathrm{~cm}$. At the target distance of $150 \mathrm{~cm}$ used in this experiment, the accuracy error was $-4.58 \%$ with precision (coefficient of variation of accuracy error) of $15.9 \%$.

Besides camera-object distance, the accuracy of the system (Kellner et al. 2004) is also influenced by the actual size of the object itself. Figure 4 shows the accuracy error of objects of increasing size at a fixed camera-object distance of $150 \mathrm{~cm}$. The accuracy error of the system is minimal for object sizes of about $60 \mathrm{~mm}$ (measurement error 0\%) and deteriorates to a maximum of 3.4\% for objects of $40 \mathrm{~mm}$ size and to $-5.6 \%$ for objects of $300 \mathrm{~mm}$.

A further accuracy error that must be considered in 3-D optics occurs when an object is not parallel to the horizontal plane but turns toward a vertical orientation. Figure 5 shows the accuracy error for an object of $80 \mathrm{~mm}$ length at increasing

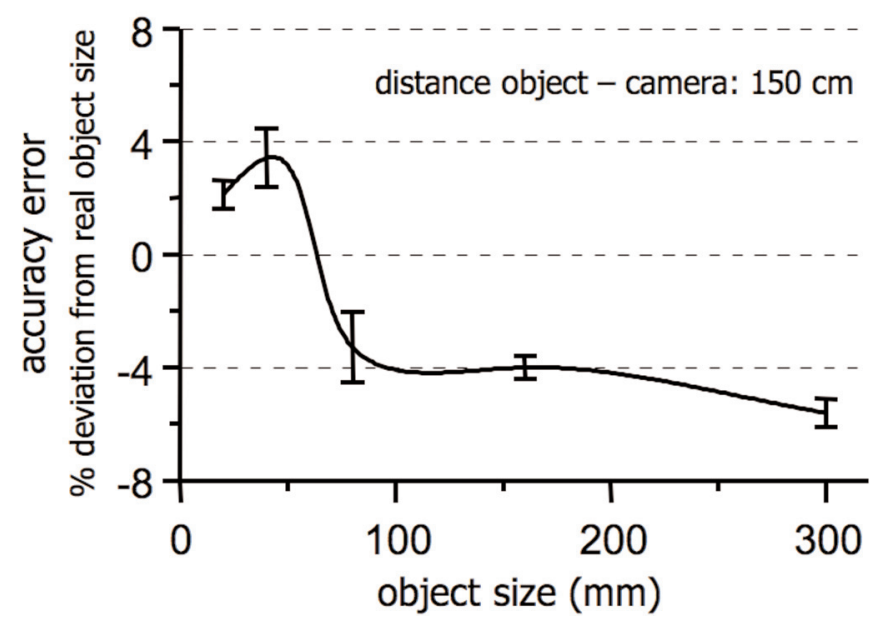

Fig. 4. Accuracy values (percent deviation between the measured size of an object and its actual size [= $100 \%]$ ) with increasing object size. Shown are mean values (connected by spline interpolation) and error bars based on 10 replicate measurements per object size. The error bars indicate the precision of the accuracy estimates and are calculated as coefficient of variation of the each accuracy value $(n=10)$. 


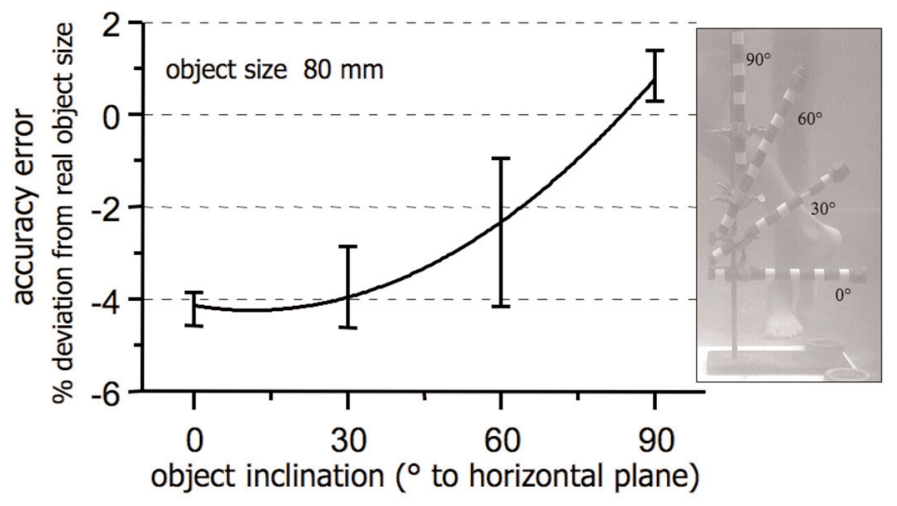

Fig. 5. Accuracy values (percent deviation between the measured size of an object and its actual size [= $100 \%]$ ) with increasing object inclination from the horizontal plane. Shown are mean values (connected by spline interpolation) and error bars based on 10 replicate measurements per inclination angle. The error bars indicate the precision of the accuracy estimates and are calculated as coefficient of variation of the each accuracy value $(n=10)$.

inclination from the horizontal $\left(0^{\circ}\right)$ to a vertical orientation $\left(90^{\circ}\right)$. With only $0.84 \%$ accuracy error and $65.5 \%$ precision, the accuracy of the system was best at an inclination angle of $90^{\circ}$ and least at $0^{\circ}$ inclination (horizontal orientation) with an accuracy error of $-4.22 \%$ (8.5\% precision).

In addition to these comparatively minor accuracy errors $(<6 \%)$, one major error occurs in all 3-D optical systems when the longitudinal (measurement) axis of an object increasingly deviates from parallel to the chip-plane of the camera toward perpendicular to the chip-plane (parallel to the optical axis). Figure 6 shows this error for the system used here with an object size of $80 \mathrm{~mm}$ at angles of $0^{\circ}$ (object is parallel to the chip-plane), $30^{\circ}, 60^{\circ}$, and $90^{\circ}$ (object is perpendicular to the chip-plane). Whereas the accuracy error is $4.12 \%$ (precision \pm

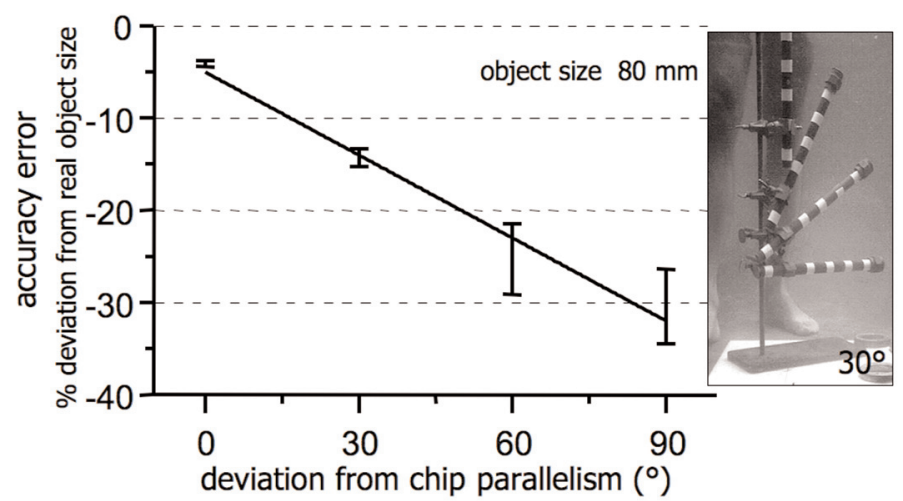

Fig. 6. Accuracy values (percent deviation between the measured size of an object and its real size $[=100 \%]$ ) with increasing deviation of an object from chip-parallelism. Shown are mean values and error bars based on 10 replicate measurements per deviation angle. Additionally, a linear regression interpolation for the relationship between the deviation angle from chip-parallelism and the mean accuracy error is plotted. The error bars indicate the precision of the accuracy estimates and are calculated as coefficient of variation of the each accuracy value $(n=10)$.
$8.01 \%)$ at $0^{\circ}$ (parallel to the chip-plane) and, therefore, well within the ranges of the above estimated errors, this accuracy error significantly deteriorates up to $30.4 \%$ (precision \pm $13.2 \%$ ) when the object becomes oriented perpendicular to the chip-plane (orientation $90^{\circ}$, parallel to the optical axis).

In our system, the relationship between the object's deviation from chip-parallelism in degree (a) and the accuracy error of the objects size (b) is linear (Fig. 6, b = -0.299 $\left.\mathrm{x} \mathrm{a}-5.042, r^{2}=0.98\right)$. This relationship can be used to correct an object's size by including the inclination angle (which can be adequately calculated by the orientation of the measured objects in the horizontal plane) in a correction algorithm. For the system used here, and for an object of $80 \mathrm{~mm}$ long, this correction algorithm was real size $=100$ $(100+c)^{-1} \times$ measured size, with $c$ being the linear error function of Fig. 6 ( $c=$ error function $=-0.229 \times$ measured deviation from chip-parallelism - 5.042).

The precision of the system at the target distance of $150 \mathrm{~cm}$ was high with an overall coefficient of variation of the accuracy of $<10 \%$ in most measurements. Exceptions include the precision value of $65.5 \%$, which occurred when measuring an $8 \mathrm{~cm}$ long object oriented vertically (object inclination $=90^{\circ}$ ) at a distance of $150 \mathrm{~cm}$ in front of the lens system, and an even lower precision with values of up to $200 \%$, which occurred when measuring an object of $8 \mathrm{~cm}$ length that is oriented horizontally (object inclination $=0^{\circ}$ ) at a distance of $250 \mathrm{~cm}$ from the lens system.

To estimate the impact of a lower precision of certain measurements, it is necessary to examine the precision in context with its respective accuracy. The precision value basically reflects the coefficient of variation $(\mathrm{CV})$ of a certain accuracy measurement as is calculated by the formulae:

$$
\frac{\text { accuracy }}{\text { standard deviation of the accuracy }} \times 100
$$

A precision of $100 \%$, therefore, means that the standard deviation of accuracy is numerically equivalent to accuracy itself. In most cases in this study system, the precision value was $<10 \%$, which means that the overall standard deviations of the accuracy measurements were lower than 10\%, and the measurement precision was therefore quite high.

In one case, however, the precision value was $65.5 \%$ and in another case up to $200 \%$. In the first case $(65.5 \%)$, the associated accuracy was quite high with an accuracy error of $0.84 \%$. An associated precision of $65 \%$ then means that the expected variation in this measurement is $0.55 \%$ of the measured object's size and therefore not a problem for assessment.

In the second case, the precision value was about $200 \%$ with an underlying accuracy of $-2.3 \%$. The expected variation in this accuracy measurement is therefore $2 \times-2.3 \%$, which means $4.6 \%$ of the size of the measured object. Even though this is much higher compared with all other precision values observed in this assessment, it shows that the system is able to determine the real size of an $8 \mathrm{~cm}$ fish that is swimming 

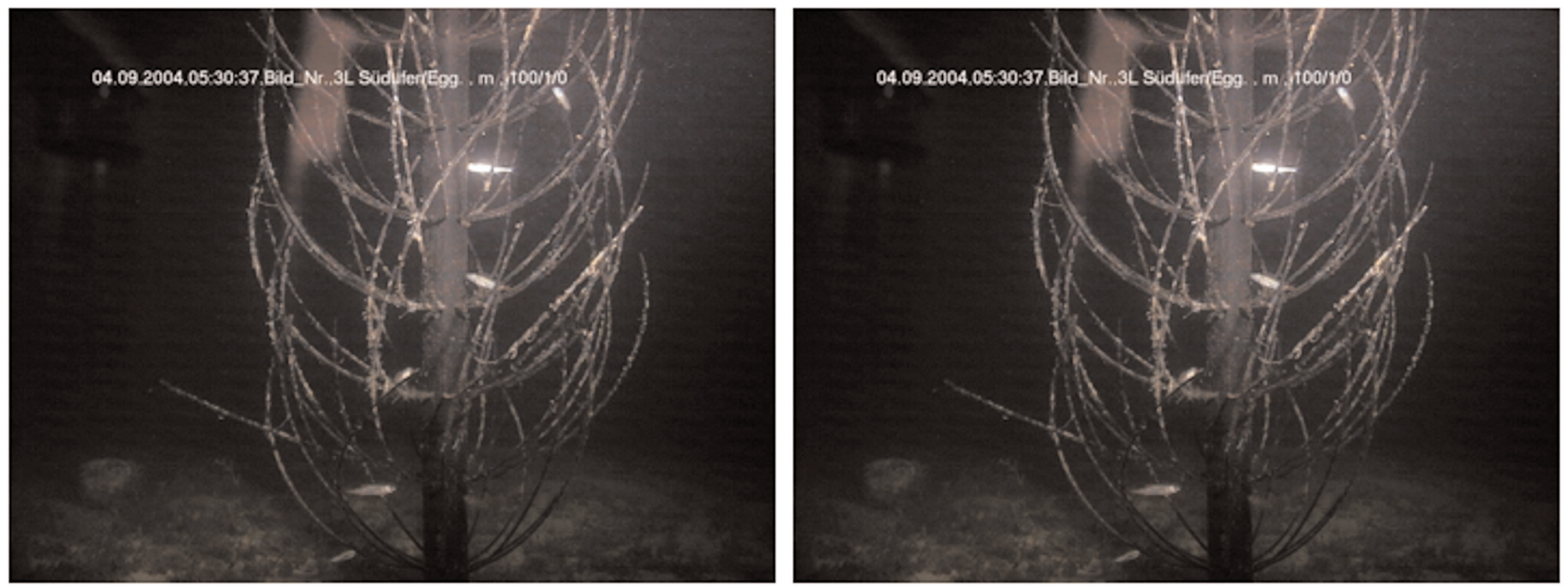

Fig. 7. Stereoscopic image pair of the artificial structure type 1/2 (see text), taken 4 September 2005 at 05:30 h. A total of six age-class 0 perch were classified as associated to the structure. Three-dimensional standard length measurements with the software-package "RemSoft1" revealed an average standard length of the fish of $5.1 \mathrm{~cm} \pm 0.8 \mathrm{SD}$.

parallel to the chip plane at a distance of $250 \mathrm{~cm}$ with a deviation of at most $5 \%$ of its real size.

In our assessment at a target distance of $1.5 \mathrm{~m}$, precision was much lower, at $15.9 \%$, and an underlying accuracy of $-4.58 \%$. These errors for the size of measured objects are, in our opinion, very acceptable for an in situ, noninvasive measurement procedure.

Because both accuracy and precision of the system were sufficiently high at the target distance of $150 \mathrm{~cm}$-with measurement deviations (accuracy) no more than 5\% from the object's length and measurement errors (precision) no more than $1 \%$ from the object's size-these measuring errors was not corrected in this assessment. However, an object's deviation from chip-parallelism $\left(^{\circ}\right)$ was corrected using the previously mentioned algorithm for real length.

In situ fish assessments-For the assessment performed here, all fish within a distance of $1 \mathrm{~m}$ from an artificial structure were classified as associated with this structure and included in the analysis. If less than 10 fish were counted, the standard lengths of all individuals were measured. If more than 10 fish were found per structure, all fish were counted but a sub-sample of 10 per species and size class were randomly selected and measured for standard length.

During our study, 4320 pictures (2160 stereoscopic image pairs) were made from the five exposed artificial structures and the control site. In Fig. 7, raw 3-D image pairs from the 2/3 structure, taken on 4 September 2005 at 05:30 h, are shown. At this sample, six age-class 0 perch with a mean total length $5.1 \pm 0.8 \mathrm{~cm} \mathrm{SD}$ were classified as associated to the structure.

Averaged over the entire sampling period, 14.3 fish (q25 = 5.5; q75 = 27.9) were counted per structure and day (Fig. 8). Of these fish, $86 \%$ were perch (Perca fluviatilis L.), $4 \%$ ruffe
(Gymnocephalus cernuus L.), and another 10\% could not be identified to species level and were classified as unidentified.

Considering the total number of fish counts $(n=996)$ over the sampling period (10 August to 6 October 2004), fish were detected in 145 of 4320 image pairs, which is $6 \%$ of all samples. This highlights the general problem of sampling complex structures with respect to the associated fish community. Pratt et al. (2005) and Brickhill et al. (2005) indicate that many studies failed to detect significant structure-fish relationships due to a lack of temporal resolution in the sampling procedure. Our study strongly supports this assumption. Our sampling frequency was $0.5 \mathrm{~h}$ throughout the day over a period of $2 \mathrm{mo}$, and we had samples in only $6 \%$ of the records. Assuming a realistic sampling frequency of once per week when

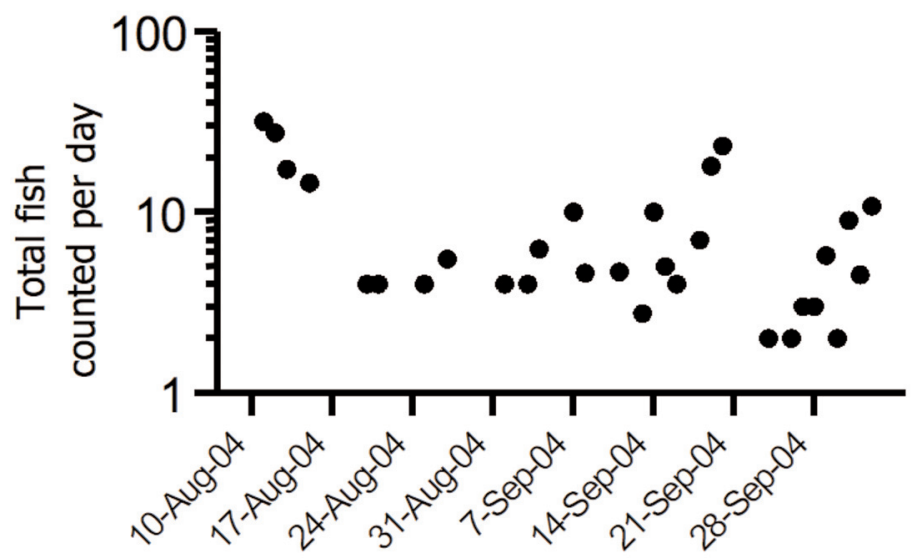

Fig. 8. Total fish abundance per day of the experiment counted by the 3-D optic of the underwater observatory. Fish were counted only when classified as associated with any of the structures. (For a detailed description of the classification procedure, "associated to a substratum type," please see text). 

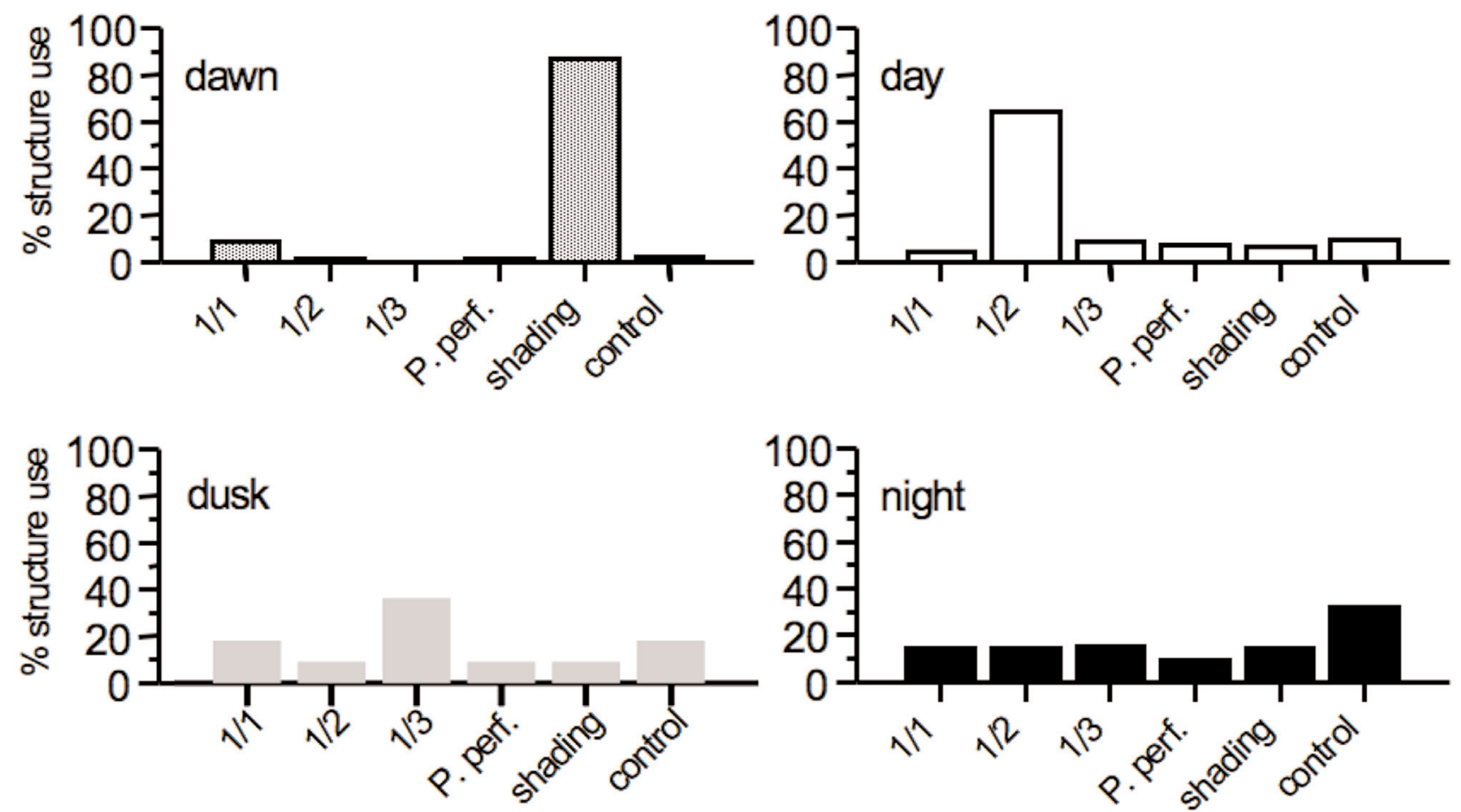

Fig. 9. Habitat preference of perch (percent fish using the five structures or the control habitat) during the daytime-phases day, dusk, night and dawn. Habitat preference data are calculated as the relative proportion of fish using one structure compared with the total abundance of fish observed at all structures.

using classical sampling devices such as pop-up nets or enclosure-removal techniques, the sample occurrence (proportion of non-zero catch), as well as the sample volume (number of fish per CPUE), would most certainly have been too low to detect any relevant pattern.

The high image sampling frequency did, however, enable us to analyze fish abundance, size class composition, and shoaling behavior (swimming direction synchronization) of the structure-associated fish community, separately for four time periods: day, dusk, night and dawn (defined by the nautical nomenclature; http://www.calsky.com/cs.cgi/Calendarhttp:// www.calsky.com/cs.cgi/Calendar?).

Figure 9 shows the structure preference of the fish separately for the four phases. During dawn, the fish showed a significant preference for the overhead cover area $(\mathrm{G}=51.9$, $\mathrm{df}=5$, $P<0.05)$ whereas during the day, they shifted to the $1 / 2$ density structure and significantly preferred this structure over all others ( $\mathrm{G}=14.7, \mathrm{df}=5, P<0.05)$. This significant pattern in structure preference ceased during dusk and at night when no significant preference for any of the five structures, or the control site, was found, and the fish used all structure types more or less uniformly (Fig. 9-dusk: $\mathrm{G}=4.18$, $\mathrm{df}=5, P>0.05$; night: $\mathrm{G}=3.53, \mathrm{df}=5, P<0.05$ ).

In addition to analysis of structure preferences, the stereooptical system allowed a detailed analysis of the length frequency distribution of the fish. Integrated over the entire sampling period, most of the perch that used the structures were age-class 0 , but older fish, up to a total length of $230 \mathrm{~mm}$, were also observed (Fig. 10).

The average total lengths of the fish at the different structures were, however, significantly different. Figure 11 shows the structure and phase-related residuals of the mean fish lengths calculated for all structures and phases of the day, on a daily basis.

Integrated over all phases of the day, significantly larger fish were observed at the $1 / 2$ density structure, intermediate

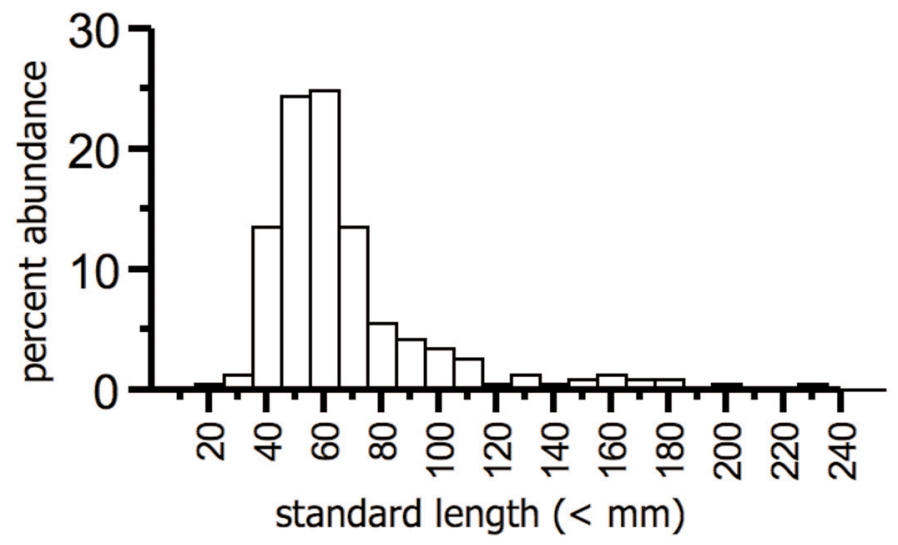

Fig. 10. Relative length (SL): frequency distribution of all fish observed at the five structures and the control site integrated over the entire sampling period. 

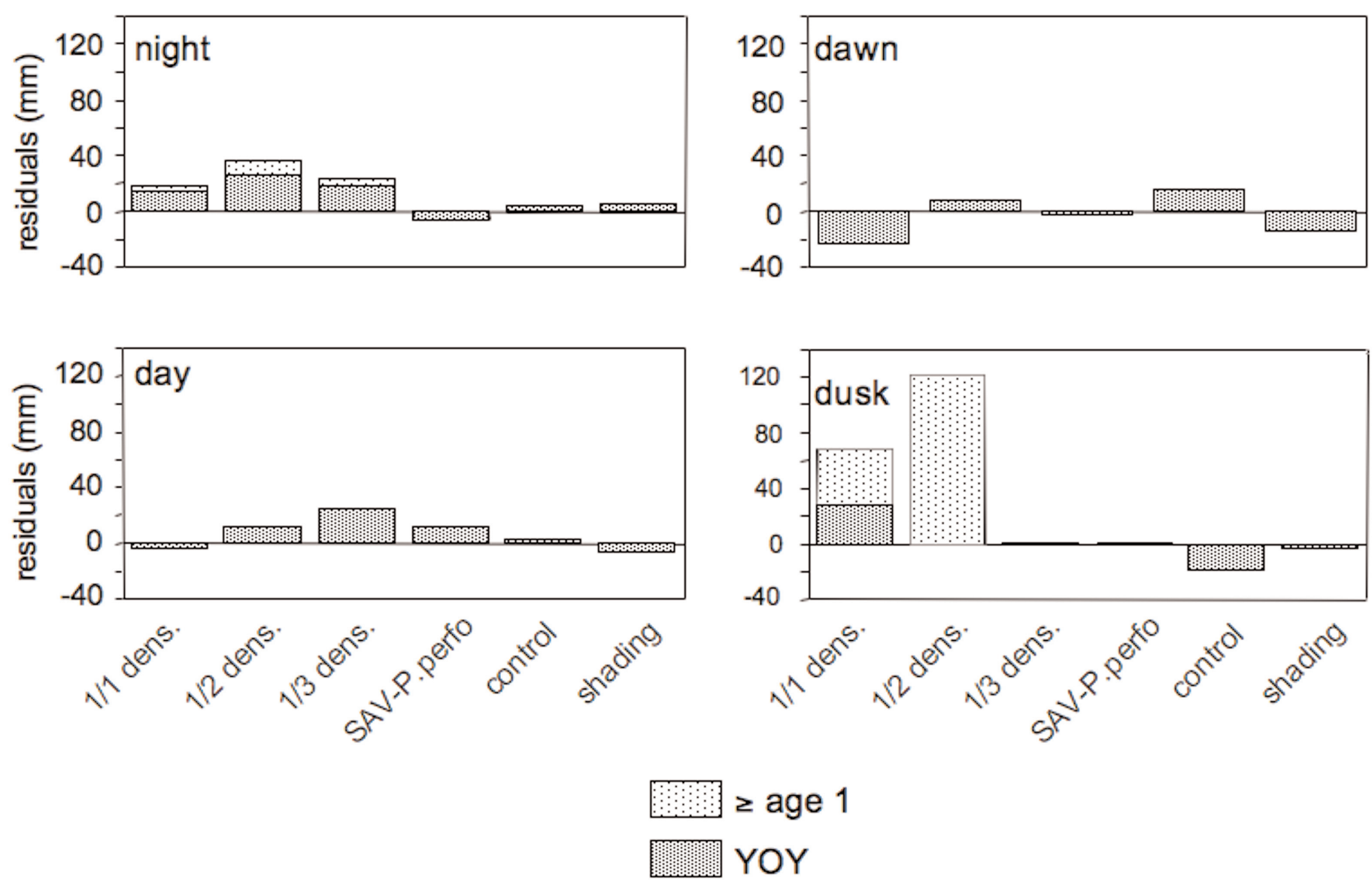

Fig. 11. Average residuals of standard lengths of the fish at the different structures and phases of the day. Shown are mean residuals based on the average fish standard length integrated over all structures and phases of the day over the entire sampling period.

fish sizes at the $1 / 1,1 / 3$, and overhead cover structures, and significantly smaller fish were found at the control site and the SAV site (ANOVA main effects: $\mathrm{df}=5, \mathrm{~F}=3.73, P=0.004$; interaction phase $\times$ structure: $\mathrm{df}=14, \mathrm{~F}=1.71, P=0.064)$. A detailed length-frequency analysis of the fish at the different structures revealed that these size differences were mainly explained by larger perch (age: 1 and older), which disproportionally used the $1 / 2$ density structure, especially during dusk and night (Fig. 11). When age 1 and older perch were excluded from the analysis, no significant differences in mean size were found between the different structures (ANOVA df $=5, \mathrm{~F}=0.96$, $P=0.45)$.

Differences in average length of the perch were, however, also found between the four phases of the day (Fig. 11). Residual analysis revealed that average fish size during dusk was significantly larger compared with the day (ANOVA df $=3$, $\mathrm{F}=3.83, P=0.012$ ), and intermediate sizes were observed during night and dawn. As for the size-distribution at the different structures, these differences in average length were explained mainly by different age-classes that used the structures at different times of day. Excluding age 1 and older fish from the analysis revealed no more significant differences in fish size at the five structures and control site between the different phases of the day (ANOVA $\mathrm{df}=3, \mathrm{~F}=0,079, P=0.97$ ).

A fish's orientation on the horizontal plane at the different structures was also analyzed. To categorize the swimming direction of a single fish, a $360^{\circ}$ orientation circle in the horizontal plane was divided into four $90^{\circ}$ windows with bisecting orientation lines at $0^{\circ}$ (parallel to the shoreline), $90^{\circ}$ (toward the pelagic zone), $180^{\circ}$ (parallel to the shoreline), and $270^{\circ}$ (toward the littoral zone). Figure 12 shows the cumulative orientation vectors of the fish as polar-plots classified according to the swimming angles $0^{\circ}, 90^{\circ}, 180^{\circ}$, and $270^{\circ}$, with one fish having a standardized vector length of 1 . Thus, the length of the vectors represent the average proportion of fish during dawn, day, dusk or night swimming in one of the four main directions. Integrated over all structures, including the control site, significant but contrary orientation vectors were observed during dawn and dusk. During dawn, the fish were significantly oriented in a $90^{\circ}$ direction (LR-Chi ${ }^{2}=23.38 \mathrm{df}=3, P<0.01$ ) indicating a migration toward the pelagic zone. In contrast, during dusk, most fish were oriented toward $270^{\circ}$, indicating a return to the littoral zone $\left(\mathrm{LR}-\mathrm{Chi}^{2}=9.98, \mathrm{df}=3, P<0.05\right)$. No significant orientation vectors were found during day and night. 

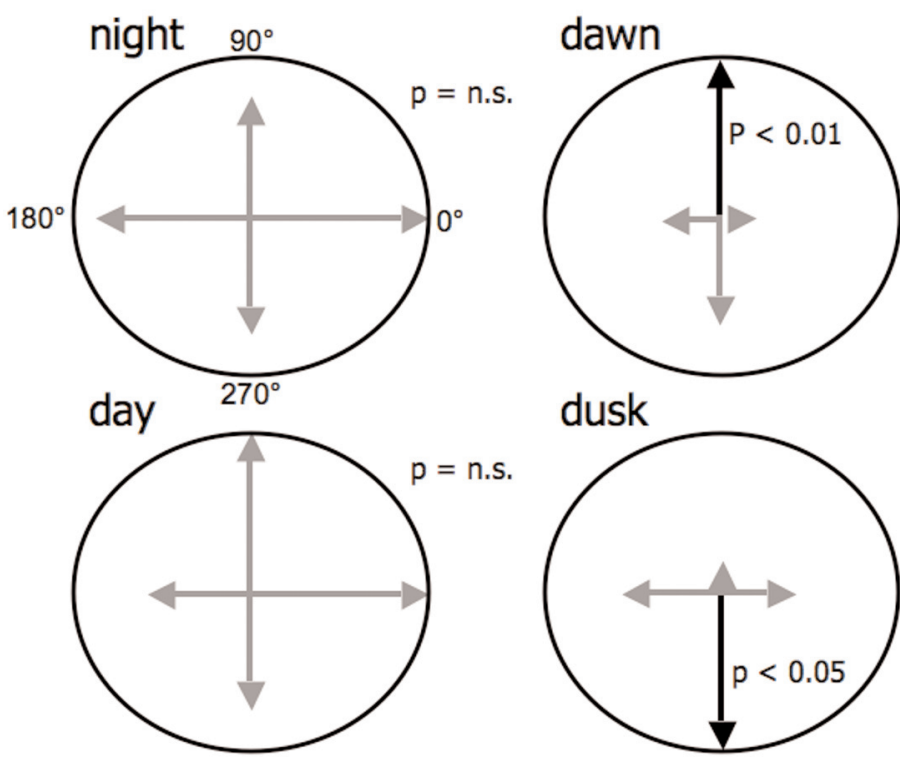

Fig. 12. Cumulative relative orientation vectors of the fish classified in the swimming angles $0^{\circ}, 90^{\circ}, 180^{\circ}$, and $270^{\circ}$, with one fish having a standardized vector length of 1 . Therefore, the lengths of the vectors represent the average proportions of fish during dawn, day, dusk, or night swimming in one of the four main directions. Zero degrees refers to an orientation of the fish toward the shoreline, $90^{\circ}$ and $270^{\circ}$ to an orientation parallel to the shoreline, and $180^{\circ}$ as swimming toward the pelagic zone.

\section{Discussion}

In contrast to terrestrial and landscape ecology where 3-D imaging is fully accepted as a method for studying complex species-habitat interactions (Lucas et al. 2002; Möller 2002; Sheng et al. 2001; Silbernagel and Moeur 2001), this type of analysis has not yet become established in aquatic ecology (but see Laurel et al. 2005; Sheng et al. 2001; Brickhill et al. 2005.) Pratt and colleagues (2005) only recently outlined the need for more sophisticated techniques, including in situ optics, for the quantitative study of fish communities in structurally complex habitats, e.g., to achieve a better estimation of the net-effects of artificial reefs on fish production in freshwater or marine systems. However, even though a variety of portable and stationary underwater imaging systems have been developed over recent years, only a few studies have so far included 3-D imaging techniques in their field studies (Aoki et al. 1986; Boisclair 1991; Dill et al. 1981; Harvey and Shortis 1996; Watson et al. 2005). Several reasons may be responsible for the lack of 3-D imaging in aquatic research. A major handicap is most certainly the cost-benefit ratio of most commercially available 3-D image systems. These systems are normally designed for use in terrestrial landscape-ecology and are therefore (i) equipped with expensive-because optically calibrated-lens systems for long-distance observations and (ii) used as analog systems, with specific film spools in order to take several hundred single pictures without changing the magazine.
Our study, as well as the early studies of Krohn and Boisclair (1994) and Klimley and Brown (1983), show that optically calibrated lens systems are not necessary to achieve adequate length measurement in aquatic studies, where the objects are normally within only a few meters of the camera system.

High-end digital cameras with state-of-the-art (noncalibrated) lens systems and sufficient resolution are available today even on the consumer market at affordable prices. Furthermore, for most of these systems, appropriate communication protocols are available to set up adequate remote control software by standard microprocessor technology via underwater cable and surface WLAN connection. This rapid development of digital photography over the last few years has therefore overcome most of the restrictions in aquatic imaging technology given by analogous systems. In this context, the most important advantages of digital versus analog photography in underwater imaging are certainly (i) the possibility of downloading pictures without recovering the system, (ii) the option to calibrate two cameras in 3-D underwater housing online to ensure parallelism of the two optical axes in the horizontal and vertical planes (Klimley 1980), and (iii) the possibility of synchronizing two still cameras using appropriate software to ensure the temporal synchronization of a pair of stereo photos. Based on these advantages of digital over analog imaging technology, several system and handling improvements have become possible that have not been available hitherto.

The system presented here allows a high sampling frequency at almost unrestricted exposure duration in a particular habitat and also allows the sampling of different habitats sequentially, e.g., in a transect line approach. As Pratt et al. (2005) already assumed, this modified set-up significantly enhanced our power of discrimination for the patterns of habitat use and enabled us to reveal significant preferences of fish for certain structures, as well as changes in these preferences over the diel cycle. The use of a high-resolution 3-D optical approach additionally allowed examination of size, and therefore, age class discrimination in fish-habitat patterns over the diel cycle.

Our results showed this technology to be highly valuable in addressing the habitat preferences and diel migration patterns of perch in Lake Constance. Furthermore, it supports the statement of Pratt et al. (2005) that in situ imaging techniques and corresponding image sampling protocols, when properly designed and technically not overloaded, can be useful tools with which to address temporal aspects of species-habitat relationships that can hardly be addressed by conventional sampling methods. Our study further showed that permanent, remotely controlled underwater observatories may be useful not only in marine, but also in freshwater ecosystems, and can be even cheaper than conventional sampling gear or standard echo-sounding equipment for fish surveys. A rough comparison of costs of the system that we used in this study arrived at 
$4180 €$ for the entire system: $1500 €$ for two digital, consumer, still cameras including flash and Web cam, $1000 €$ for the fabrication of a V2A-watertight housing and rack, $800 €$ for electronic equipment, including a USB-splitter to simultaneously trigger the two cameras, $180 €$ for a $150 \mathrm{~m}$ long underwater usable cable and, additionally, $700 €$ for one 800 $\mathrm{MHz}$ IBM computer including remote control software and WLAN technology system. Compared with either standard echosounder equipment able to estimate sizes and densities of fishes in shallow littoral areas, or to other methods for sampling fish at half-hour intervals over $2 \mathrm{mo}$, the system used here is certainly cost competitive. A further advantage of in situ optical systems over echo-sounder technology is the possibility of identifying fish to species level. This is especially important for littoral areas where the species diversity is normally higher than in pelagic systems and fish aggregations cannot be assigned to a single species with a high probability.

\section{Comments and recommendations}

In contrast to classical net sampling methods, both optical and acoustic systems have the advantages of being less laborintensive in the field, able to operate in a stationary position over $24 \mathrm{~h}$, and much less invasive in terms of fish killed to measure abundance and size at temporally high resolution. However, this advantage also may be a disadvantage in that none of the technical sampling methods (optic or acoustic) are capable of obtaining data such as stomach contents or detailed ageclass estimation, e.g., by otolith analysis. Therefore, technical sampling methods such as in situ optic or acoustic assessments should, if possible, be accompanied by classical net sampling, which then, however, have to be taken at a much lower frequency to save labor, time, and aquatic resources.

The calibration experiments revealed that, in our setup, the system-related measurement deviation (accuracy) and the measurement error (precision) were sufficiently low to remain uncorrected, except in the case of object deviations from chip parallelisms. However, these measurement errors should have to be considered when objects are measured in distances greater than about $250 \mathrm{~cm}$, as well as when objects are measured that are significantly smaller than the fish measured here. The integration of these measurement errors as correction algorithm for system accuracy, especially as error term for system precision, should be included as the next step in the length-calculation algorithm in the RemSoft analysis software for 3-D image analysis.

Another possible bias when using observatory technology for studying temporal and spatial patterns in fish abundance and species diversity in a certain habitat is the observatory itself. Because the construction of an underwater observatory is normally focused on constructive necessities rather than on structural camouflage, observatories do act as structure itself. This may become a problem, especially in habitats with comparatively low structural complexity, because the observatory itself may attract aquatic organisms and thus producesassessment artifacts.
Unfortunately, there are few studies available that specifically focus on such interactions between observatories and the environment, especially the problems caused by observatoryinduced artifacts in habitat assessments. For deep-sea lander systems, Parker et al. (2003) noted that, e.g., autonomous deep-sea lander systems significantly interact with the substrate in varying time scales, and that thorough assessment of these interactions and their effects on the results of these systems are fundamentally important in estimating data quality. The study of these interactions and the impact of these systems, therefore, have to be addressed in the future to ensure representative and artifact-free data.

Similar observations as described by Parker et al. (2003), i.e., that the observatory itself acts as structure and attracts fish and other organisms were made also in this study. After 3 mo exposure, especially bivalves (i.e., Dreissena polymorpa) heavily colonized almost all metal surfaces of the observatory. Additionally, crayfish and small benthic fish, such as juvenile burbot, were observed from time to time associated to the observatory itself, when the underwater set-up was visually inspected and maintained by scientific divers of the University Konstanz.

However, in contrast to the above-mentioned structurally poor deep sea habitats, structural complexity as well as species abundance in littoral freshwater habitats is normally quite high. Therefore, even though a comparatively small underwater installation like our RemOs1 system does provide an additional artificial structure in such habitat, it certainly does not generate unique structure in the area that significantly deducts fish and mobile invertebrates from other structures to such an extent that structure-fish associations are significantly affected.

Another interaction with the environment, especially when using in situ optic observatory systems, may be produced by the use of artificial light. This may produce a severe bias in species abundance assessments when permanent illumination or high frequency flash illumination is applied, because most mobile organisms are either positively or negatively phototactic and are, therefore, either attracted or repelled by artificial light. In our study, we decided to keep the image and flash illumination frequency as low as possible (on flash illumination per $30 \mathrm{~min}$ ) to reduce these kind of problems as much as possible. Because the observatory continuously sampled a certain structure over 6 days, and we could not detect a significant change in fish abundance or species composition during these consecutive days, we are quite sure that the flash illumination frequency used in our experimental setup had no severe effects on the fish community itself. However, this problem certainly has to be considered in higher frequency sampling with flash illumination or any kind of permanent illumination within the visible spectrum of light. We recommend that in an in situ optic study that uses flash illumination technology, researchers thoroughly review the need for higher frequency sampling in addressing a certain scientific question and adapt any flash supported in situ sampling protocol to the lowest frequency possible. 
Even though the possibilities for in situ data assessment may be further extended by remote operated in situ 3-D technology and improved analysis software, the laboratory data analysis, e.g., digital image analysis to properly identify and measure fish or other organisms on the 3-D images will become much more intensive. One major goal in the development and use of in situ optical imaging in aquatic science should therefore be the development of improved imageanalysis tools for semi- or fully automated data assessment, e.g., for 3-D image pairs. Such high-end technologies have been successfully developed in hydro-acoustics over recent decades and have already proved their usefulness. Comparable hardware and software developments, however, are still almost completely missing in hydro-optics. If such technologies and subsequent analysis tools are successfully developed in the future, autonomously working in situ optical systems can greatly extend our possibilities for sampling aquatic communities at the necessary resolution to resolve patterns at ecologically relevant temporal and spatial scales.

\section{References}

Altris, I. 2001. Carbon copy, 5.60.280 ed. <http://www.altiris. $\mathrm{com} />$

Aoki, I., T. Inagaki, and L. Van Long. 1986. Measurements of the three-dimensional structure of free-swimming pelagic fish schools in a natural environment. Am. Nat. 52:2069-2077.

Basset, C. E. 1994. Use and evaluation of fish habitat structures in lakes of the eastern United States by the USDA Forest Service. Bull. Mar. Sci. 55:1137-1148.

Bohnsack, J. A., and D. L. Sutherland. 1985. Artificial reef research: a review with recommendations for future priorities. Bull. Mar. Sci. 37:11-39.

Boisclair, D. 1991. An evaluation of the stereocinematographic method to estimate fish swimming speed. Can. J. Fish. Aquat. Sci. 49:523-531.

Brickhill, M. J., S. Y. Lee, and R. M. Connolly. 2005. Fishes associated with artificial reefs: attributing changes to attraction or production using novel approaches. J. Fish. Biol. 67: 53-71.

Cook, R. W. 1986. Underwater imaging: One user's perspective. Sea-Technol. 27:29.

Crowder, L. B., and W. E. Cooper. 1982. Habitat structural complexity and the interactions between Bluegills and their prey. Ecol. 63:1802-1813.

Diehl, S., and R. Kornijow. 1998. Influence of submerged macrophytes on trophic interactions amongfish and macroinvertebrates, p. 24-46. In E. Jeppesen, M. Sondergaard, M. Sondergaard, and K. Christoffersen [Eds.], The structuring role of macrophytes in lakes. Springer Verlag.

Dill, L. M., R. L. Dunbrack, and P. F. Major. 1981. A new stereophotographic technique for analyzing the threedimensional structure of fish schools. Env. Biol. Fish. 6:7-13.

Fischer, P. 2000. Test of competitive interactions for space between the two benthic fish species, burbot (Lota lota L.) and stone loach (Barbatula barbatula L.). Env. Biol. Fish. 58:439-446.

- 2004. Effects of intraspecific competition and predation risk in the littoral-benthic community: a case study on juvenile burbot (Lota lota). Bor. Env. Res. 9:213-225.

Harvey, E., and M. Shortis. 1996. A system for stereo-video measurement of sub-tidal organisms. Mar. Tech. Soc. J. 29: 10-22.

Johnson, D. L., R. A. Beaumier, and W. E. J. Lynch. 1988. Selection of habitat structure interstice size by bluegills and largemouth bass in ponds. Trans. Am. Fish. Biol. 117:171-179.

- and W. E. Lynch. 1992. Panfish use of an angler success at evergreen tree, brush, and stake-bed structures. N. Am. J. Fish. Manage. 12:222-229.

Kellner, R., J.-M. Mermer, M. Otto, H. Valcarcel, and H. M. Widmer. 2004. Analytical Chemistry: A modern approach to analytical science. Wiley, VCH.

Klimley, P. A. 1980. Stereo-photographic analysis. A stereophotographic technique for the determination of lengths of freeswimming sharks. CIBASCO-Transactions 6:113-137.

— and S. T. Brown. 1983. Stereophotography for the field biologist: measurement of lengths and three-dimensional positions of free-swimming sharks. Mar. Biol. 74:175-185.

Krohn, M. M., and D. Boisclair. 1994. Use of a stereo-video system to estimate the energy expenditure of free-swimming fish. Can. J. Fish. Aquat. Sci. 51:1119-1127.

Kuznetsov, V. A. 1970. Spawning grounds of some percid fishes in Sviyaga Bay, Kuybyshev Reservoir, the distribution of larvae and the effectiveness of reproduction. J. Ichthyol. 10:765-771.

Larson, E. W., and D. L. Johnson. 1986. A buoyant pop net for accurately sampling fish at artificial habitat structures. Trans. Am. Fish. Biol. 115:351-355.

Laurel, B. J., J. A. Brown, R. S. Gregory, and C. J. Laurel. 2005. A new technique to gather 3-D spatial information using a single camera. J. Fish. Biol. 66:429-441.

Lucas, R. M., J. C. Ellison, A. Mitchell, B. Donnelly, M. Finlayson, and A. K. Milne. 2002. Use of stereo aerial photography for quantifying changes in the extent and height of mangroves in tropical Australia. Wetland Ecol. Manag. 10:161-175.

Manatunge, J., T. Asaeda, and T. Priyadarshana. 2000. The influence of structural complexity on fish-zooplankton interactions: A study using artificial submerged macrophytes. Env. Biol. Fish. 58:425-438.

Möller, M. 2002. New remote sensing systems and GIStechniques for the monitoring of urban ecological processes, p. 109-117. In Manfred Ehlers (Ed), Proceedings of SPIE, The International Society for Optical Engineering. SPIE, USA.

Parker, W. R., K. Doyle, E. R. Parker, P. J. Kershaw, S. J. Malcolm, and P. Lomas. 2003. Benthic interface studies with landers. Consideration of lander/interface interactions and their design implications. J. Exp. Mar. Biol. Ecol. 285-286: 179-190. 
Persson, L., and P. Eklöv. 1995. Prey refuges affecting interactions between piscivorous perch and juvenile perch and roach. Ecology 76:70-81.

Pratt, T. C., K. E. Smokorowski, and J. R. Muirhead. 2005. Development and experimental assessment of an underwater video technique for assessing fish-habitat relationships. Arch. Hydrobiol. 164:547-571.

Priyadarshana, T., T. Asaeda, and J. Manatunge. 2001. Foraging behaviour of planktivorous fish in artificial vegetation: The effects on swimming and feeding. Hydrobiol. 442:231-239.

Rebikoff, D. 1984. Two eyes: Stereovideo and videogrammetry ROV'84. Tech. update and international perspectives. Marine Technology Society, San Diego.

Rogers, K. B., and E. P. Bergersen. 1999. Utility of synthetic structures for concentrating adult Northern pike and largemouth bass. N. Am. J. Fish. Manage. 19:1054-1065.

Sheng, Y., P. Gong, and G. S. Biging. 2001. Model-based conifer-crown surface reconstruction from high-resolution aerial images. Photogram. Eng. Remote Sens. 67:957-965.

Silbernagel, J., and M. Moeur. 2001. Modeling canopy openness and understory gap patterns based on image analysis and mapped tree data. Forest Ecol. Manage. 149:217-233.

Watson, D. L., E. S. Harvey, G. A. Kendrick, and M. J. Anderson. 2005. A comparison of temperate reef fish assemblages recorded by three underwater stereo-video techniques. Mar. Biol. 148:415-425.

Weaver, M. J., J. J. Magnuson, and M. K. Clayton. 1997. Distribution of littoral fishes in structurally complex macrophytes. Can. J. Fish. Aquat. Sci. 54:2277-2289.

Welcomme, R., and I. G. Cowx. 1998. Rehabilitation of rivers for fish. Blackwell Science.

Submitted 5 May 2006

Revised 1 April 2007

Accepted 22 April 2007 